\title{
Study on the interaction of Prodigiosin with bovine serum albumin by spectroscopic methods
}

\author{
Shu-Chao Liu ${ }^{\text {a,b,c }}$, Jing Tang ${ }^{\mathrm{d}}$, Xi-Hai Zhang ${ }^{\mathrm{a}, \mathrm{b}, \mathrm{c}}$, Yuan-Yuan Gao ${ }^{\text {a,b,c }}$, Fei Ma ${ }^{\mathrm{d}}$ and \\ Qi-yin Yang ${ }^{a, b, c, *}$ \\ ${ }^{a}$ College of Life Science, Nanjing Normal University, Nanjing, China \\ b Jiangsu Key Laboratory for Microbes and Functional Genomics, College of Life Sciences, Nanjing \\ Normal University, Nanjing, China \\ ${ }^{\mathrm{c}}$ Jiangsu Province Engineering and Technology Research Center for Microbiology, College of Life \\ Science, Nanjing Normal University, Nanjing, China \\ ${ }^{\mathrm{d}}$ Center for Analysis and Testing, Nanjing Normal University, Nanjing, China
}

\begin{abstract}
The interaction between bovine serum albumin (BSA) and Prodigiosin (PG) was investigated by UV-vis absorption, fluorescence, synchronous fluorescence, FT-IR and circular dichroism (CD) techniques. The data of UV-vis absorption and fluorescence spectra displayed that there existed interaction between PG and aromatic amino acid residues of BSA. The synchronous fluorescence and CD spectrum experiment both showed that the secondary structure of BSA changed with addition of PG. All these results revealed that the conformation and microenvironment of BSA were changed.
\end{abstract}

Keywords: Prodigiosin, bovine serum albumin, interaction, spectroscopic methods

\section{Introduction}

Prodigiosin (PG) (Scheme 1) is a red, linear tripyrrole and a member of the prodiginines, produced by some Serratia sp., Actinomycetes and a few other Bacteria [12]. Prodigiosin is classical secondary metabolites only appearing in later stages of bacterial growth [27]. Prodigiosin itself has considerable immunosuppressive activity, antibacterial, antimycotic and antimalarial activity, etc. [3,22,26], but high toxicity precludes its application as a therapeutic agent. In recent years, the research on PG aimed mainly at its potential as a drug to resist tumor in clinical practice. The US National Cancer Institute (NCI) confirmed that PG had a stronger resistibility to 57 various human cancer cells such as in vitro liver cancer transfer and primary cancer cells, moreover, PG had not obvious toxicity to nonmalignant celllines [8].

Serum albumin is the major protein constituent of blood plasma which facilitates the disposition and transportation of various exogenous and endogenous ligands to the specific targets [16]. Bovine serum

\footnotetext{
*Corresponding author: Qi-yin Yang, Jiangsu Province Engineering and Technology Research Center for Microbiology, College of Life Science, Nanjing Normal University, Nanjing 210046, People's Republic of China. Tel.: +86 2585891617 ; Fax: +86 25 85891526; E-mail: yangqiyin@163.com.
} 


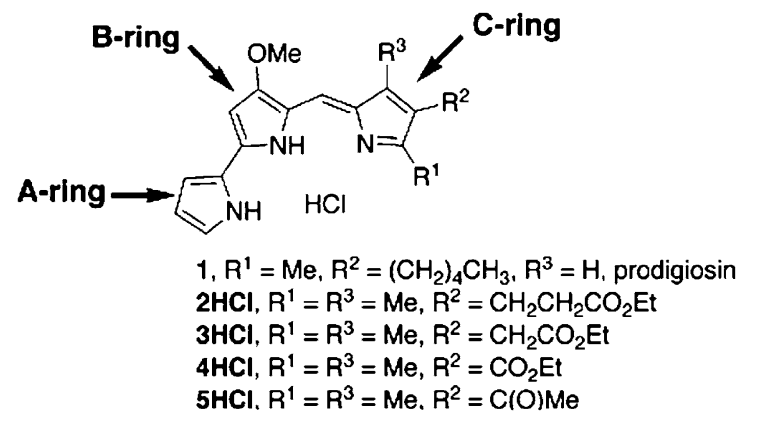

Scheme 1. The pyrrolyl dipyrrolyl methylene skeleton of prodigiosin and prodigiosus.

albumin (BSA) is one of the serum albumins, and it has been widely investigated because of its highly structural homology with human serum albumin [24]. BSA has been applied extensively in the fields of molecular biology and cell biology for its physicochemical properties are well characterized. Being the major binding protein for drugs and other physiological substances, BSA is considered as a model protein for studying protein-drug interaction in vitro. Thus, BSA have been studied extensively in the past yeas.

The study of interactions between proteins and drugs has become the hot spot in the field of life science, chemistry, and clinical medicine in recent years because most drugs undergo a greater or lesser extent of reversible binding to plasma proteins and blood cells $[15,25,28]$. The most important property of BSA is that it serves as a depot protein and transport protein for many drugs and other small bioactive molecules. Investigating on the interactions of PG with BSA can elucidate whether PG can transfer in the blood system, and provide useful information for clinical medicine.

\section{Materials and methods}

\subsection{Materials}

BSA used in this experiment was purchased from Sigma (St. Louis, MO, USA) and used without further purification. PG (purity $>88 \%$ ) was produced by Serratia marcescens and purified according to the procedures described previously [17]. All other reagents used were of analytical grade and used without further purification. All aqueous solutions were prepared using the deionized water.

\subsection{Apparatus and methods}

The UV-vis absorption spectra were recorded on a Varian Cary 5000 spectrophotometer equipped with $1.0 \mathrm{~cm}$ quartz cells with $1.0 \mathrm{~nm}$ path length. The range of wavelength was from 200 to $700 \mathrm{~nm}$.

Fluorescence spectroscopy was executed with a Perkin-Elmer LS-50B fluorescence spectrophotometer equipped with a $1.0 \mathrm{~cm}$ quartz cell and a thermostatic bath. Experiment investigation of fluorescence quenching mechanisms was carried out at different temperatures, such as at 298 and $310 \mathrm{~K}$. The fluorescence intensity of protein at $340 \mathrm{~nm}$ was determined under the excitation at wavelength of $280 \mathrm{~nm}$.

FT-IR measurements were carried out on a Nicolet Nexus 670 FT-IP infrared spectrometer. All spectra were taken via the Attenuated Total Reflection (ATR) method with resolution of $4 \mathrm{~cm}^{-1}$ and 512 scans. Spectra processing procedures: spectra buffers were collected at the same conditions. Then, the 
absorbance of the buffer solution was subtracted from the spectra of the sample solution to obtain the FT-IR spectra of the protein.

CD measurements were taken with an Applied-Photophysics Chriascan spectrophotometer in a $1.0 \mathrm{~cm}$ quartz cell at room temperature. Spectra were scanned with $1.0 \mathrm{~nm}$ spectral bandwidth and $0.5 \mathrm{~nm}$ step resolution. Measurements were recorded in the range of 190-260 nm.

\section{Results and discussion}

\subsection{UV/vis absorption studies}

UV/vis absorption measurement is a very simple method to explore the structural change and to know the complex formation [13]. Figure 1 showed the UV/vis absorption spectra of BSA from 200 to $700 \mathrm{~nm}$ in the presence of various PG concentrations. As can be seen from Fig. 1, BSA has a strong absorption peak at $278 \mathrm{~nm}$, which represented the characteristic absorption band of Trp residues. The absorption of BSA at $278 \mathrm{~nm}$ increased obviously with the addition of PG, the reason stem from the interaction between PG and aromatic amino acid residues in BSA molecular external, the results indicated that the Oxygen groups in PG interacted with the oxygen groups or nitrogen groups in the amino acid residues of BSA peptide chain, which led to the microenvironment of BSA changed in the interaction.

In addition, the absorption peak in $550 \mathrm{~nm}$ was the characteristic absorption peak of PG.

\subsection{Fluorescence studies}

For macromolecules, fluorescence measurements can give some information about binding of small molecules to proteins. It is proverbial that the fluorescence of BSA comes from the tyrosine, tryptophan, and phenylalanine residues. BSA has a strong fluorescence emission at $340 \mathrm{~nm}$ on exciting at $280 \mathrm{~nm}$ [2].

BSA has three fluorophores, which are tryptophan (Trp), tyrosine (Tyr) and phenylalanine (Phe). The intrinsic fluorescence of BSA when excited at $295 \mathrm{~nm}$ is mainly due to the presence of the two Trp residues. However, the intrinsic fluorescence of BSA when excited at $280 \mathrm{~nm}$ is mainly attributed to the presence of the Trp and Tyr residues.

The fluorescence quenching spectra of BSA induced by PG in different temperatures were showed in Figs 2 and 3. The curve of the BSA fluorescence quenching by PG was not linear, namely, the quenching

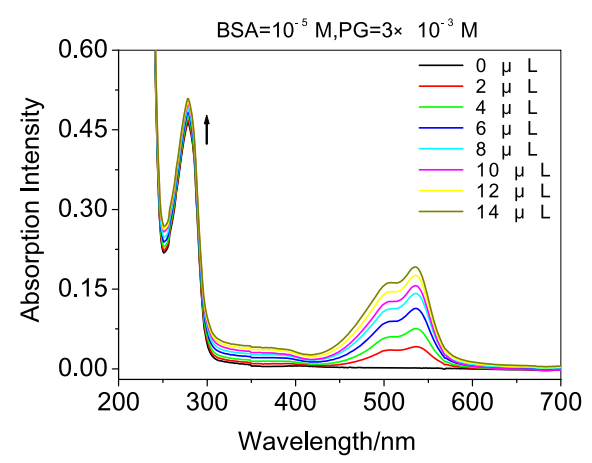

Fig. 1. Absorption spectra of BSA and BSA-PG systems. BSA concentration was $1.0 \times 10^{-5} \mathrm{~mol} / \mathrm{l}$. PG concentration was $3.0 \times 10^{-3} \mathrm{~mol} / \mathrm{l}$. (Colors are visible in the online version of the article; http://dx.doi.org/10.3233/SPE-2012-0561.) 


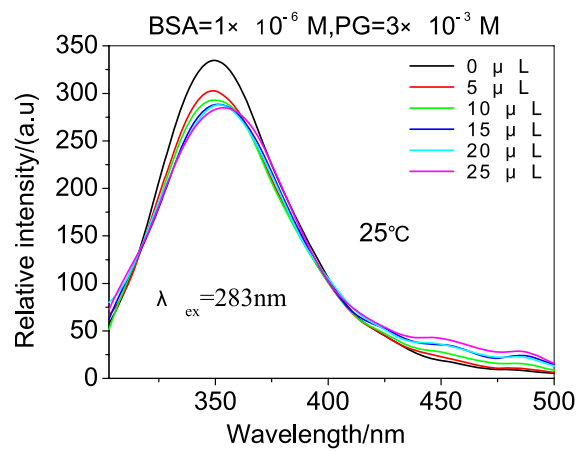

(a)

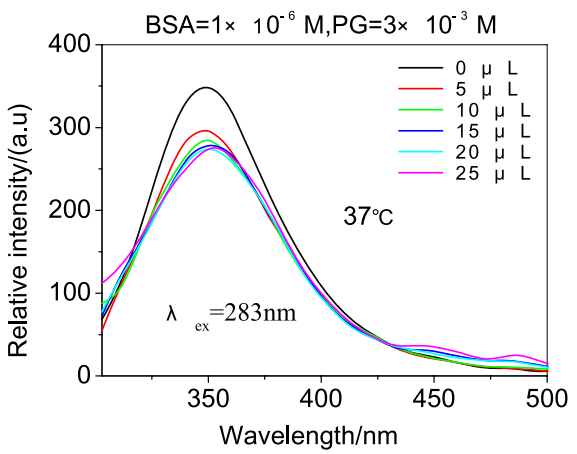

(b)

Fig. 2. Fluorescence spectra of BSA and of BSA treated with different content of PG at $\lambda_{\mathrm{ex}}=280 \mathrm{~nm}$. BSA concentration was $1.0 \times 10^{-6} \mathrm{~mol} / \mathrm{l}$. PG concentration was $3.0 \times 10^{-3} \mathrm{~mol} / \mathrm{l}$. (a) $T=298 \mathrm{~K}$, (b) $T=310 \mathrm{~K}$. (Colors are visible in the online version of the article; http://dx.doi.org/10.3233/SPE-2012-0561.)

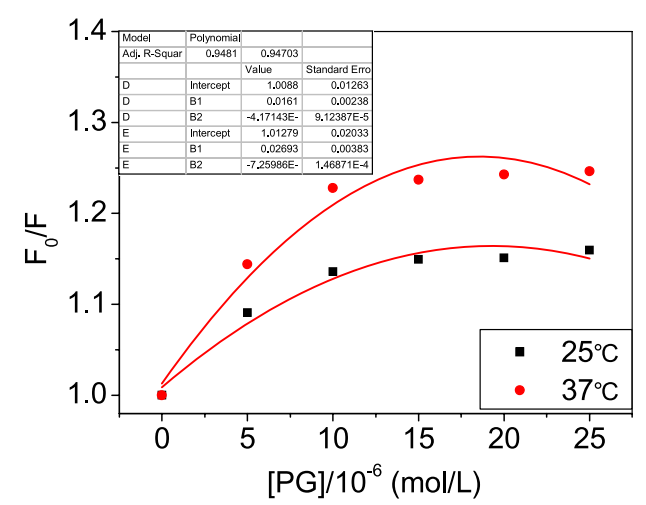

Fig. 3. The Stern-Volmer plot for the quenching of BSA by PG at different temperature. (Colors are visible in the online version of the article; http://dx.doi.org/10.3233/SPE-2012-0561.)

type was not single static or dynamic quenching, which was unmatched the Stern-Volmer equation. The quenching type would be combined quenching (both static and dynamic), it was in line with secondorder polynomial S-V equation.

\subsection{FT-IR studies}

FT-IR spectroscopy is one of the valuable tools to monitor the conformational changes at the secondary structure level in proteins that span different components such as $\alpha$-helix, $\beta$-sheet, turns or coil, and intraand intermolecular aggregates [1,4,5,9,10,14,20,21,23].

In order to determine the effect of PG on the secondary structure of BSA, FT-IR spectra of BSA and mixture of BSA and PG are measured.

Infrared spectra of proteins exhibit a number of so-called amide bands, which represent different vibrations of the peptide moieties. Of all the amide bands of the protein, the amide I ranging from $1600-1700 \mathrm{~cm}^{-1}$ (principally $\mathrm{C}=\mathrm{O}$ stretching vibration) and the amide $\mathrm{II} \sim 1548 \mathrm{~cm}^{-1}$ (C-N stretching vibration with $\mathrm{N}-\mathrm{H}$ bending vibration mode) have been widely used as typical ones [18]. They both have relationship with the secondary structure of the protein. 
Figure 4 was the FT-IR spectra of free BSA and BSA-PG. It is apparent that in the presence of PG, the band of amide I of BSA shifted from 1647 to $1645 \mathrm{~cm}^{-1}$ and amide II shifted from 1548 to $1550 \mathrm{~cm}^{-1}$. Simultaneously the shapes of their peaks all became broader. The above results represented that PG interacted with the amide groups in protein polypeptides and caused the rearrangement of the polypeptide carbonyl hydrogen bonding network.

\subsection{Investigation on the conformation of BSA}

To examine the effect of PG on the conformation of BSA, CD spectra and synchronous fluorescence spectra were measured. hydrogen bonding network.

\subsubsection{CD spectra}

To further investigate the structural changes of BSA were associated with PG, CD spectra of BSA were recorded with and without the addition of PG. Figure 5 expressed CD spectra for the BSA samples in the absence and presence of PG. The CD spectrum of BSA exhibit two negative bands in the UV region at 208 and $220 \mathrm{~nm}$, characteristic of an $\alpha$-helix structures of protein [6]. A reasonable explanation that the negative peaks between 208 and $209 \mathrm{~nm}, 222$ and $223 \mathrm{~nm}$ are both contributed by $n-\pi^{*}$ transition for the peptide bond of $\alpha$-helix [11]. Figure 6 showed that there was a slight reduction in both of the positive and typical negative peaks in the presence of PG, indicating a slight decrease of the $\alpha$-helix structures of BSA. And the loss of the $\alpha$-helix revealed that PG molecules associated with the amino acid residues of the main polypeptide chain of protein and destroyed their hydrogen bonding networks [30, 31]. Moreover, the CD spectra of BSA in the absence and presence of PG were similar, which interpreted that the structure of BSA after addition of PG was still predominantly $\alpha$-helix. The addition of PG to BSA induced only a decrease with the intensities of the peaks and without any evident shift of the peaks, revealed the slight changes in the protein secondary structure by PG.

\subsubsection{Synchronous fluorescence spectra}

Synchronous fluorescence spectroscopy is one of the commonly used methods to study the conformations of proteins which were introduced by Lloyd [19]. It involves simultaneous scanning of the excitation and emission monochromators while maintaining a constant wavelength interval between them. Synchronous fluorescence spectroscopy gives information about the molecular environment in the

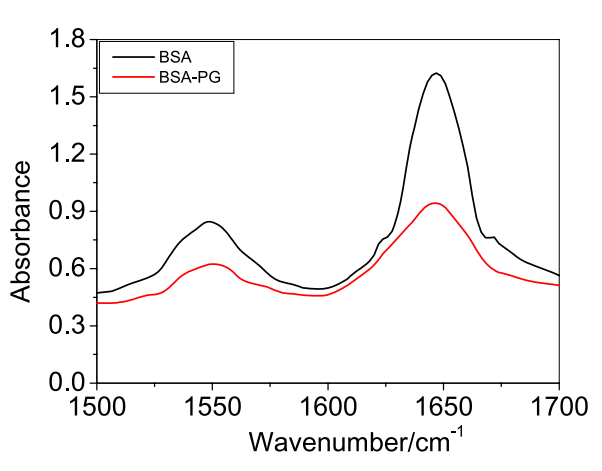

Fig. 4. FT-IR spectra of BSA and BSA-PG. (Colors are visible in the online version of the article; http://dx.doi.org/ 10.3233/SPE-2012-0561.)

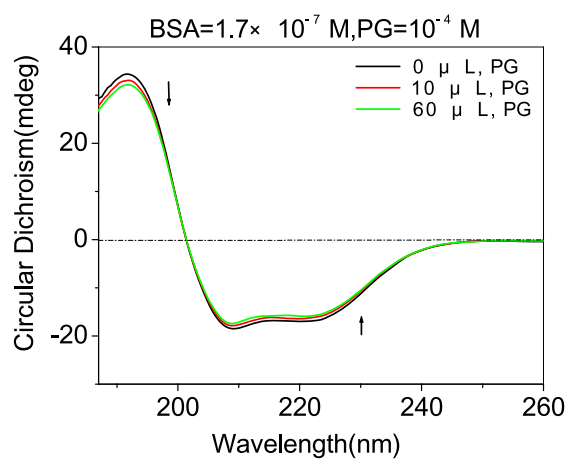

Fig. 5. The CD spectra of the BSA in absence and presence of PG. BSA concentration was $1.7 \times 10^{-7} \mathrm{~mol} / \mathrm{l}$. $\mathrm{PG}$ concentration was $1.0 \times 10^{-4} \mathrm{~mol} / \mathrm{l}$. (Colors are visible in the online version of the article; http://dx.doi.org/ 10.3233/SPE-2012-0561.) 


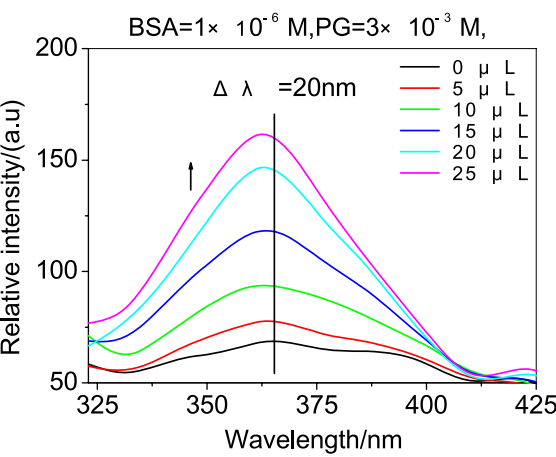

(a)

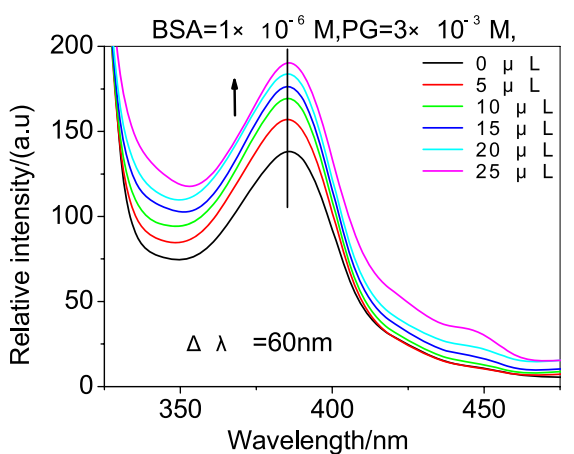

(b)

Fig. 6. The synchronous fluorescence spectra of the BSA in absence and presence of PG. BSA concentration was $1.0 \times 10^{-6} \mathrm{~mol} / \mathrm{l}$. PG concentration was $3.0 \times 10^{-3} \mathrm{~mol} / \mathrm{l}$. (a) $\Delta \lambda=20 \mathrm{~nm}$, (b) $\Delta \lambda=60 \mathrm{~nm}$. (Colors are visible in the online version of the article; http://dx.doi.org/10.3233/SPE-2012-0561.)

vicinity of the chromosphere molecules and has several advantages, such as sensitivity, spectral simplification, spectral bandwidth reduction and avoiding different perturbing effects [13]. Yuan et al. [29] suggested a useful method to study the environment of amino acid residues by measuring the possible shift in wavelength emission maximum $\lambda_{\max }$, the shifts in position of emission maximum corresponding to the changes of the polarity around the chromosphere molecule. When the D-value $(\Delta \lambda)$ between excitation wavelength and emission wavelength was stabilized at 15 or $60 \mathrm{~nm}$, the synchronous fluorescence give the characteristic information of tyrosine residues or tryptophan residues, respectively [7].

The Synchronous fluorescence spectra of BSA interact with the various concentrations of PG were shown in Fig. 6. It can be seen that there was not the Synchronous fluorescence spectra of PG in BSA solution, the fluorescence emission peak of BSA was at $363 \mathrm{~nm}$ when $\Delta \lambda$ was $20 \mathrm{~nm}$, which was the emission peak of tyrosine residues in BSA, the fluorescence emission peak of BSA was at $386 \mathrm{~nm}$ when $\Delta \lambda$ was $60 \mathrm{~nm}$, which was the emission peak of tryptophan residues in BSA.

When different concentration of PG solution titrated into a fixed concentration of BSA, remarkable changes of the fluorescence emission peak of BSA were observed, and the emission peak of tyrosine residues have a blue shift and the fluorescence intensity decreased. Whereas a slight red shift of the emission peak of tryptophan residues was observed, the fluorescence intensity decreased also.

In comparison of the two emission peaks indicated that there was a greater influence to the fluorescence emission peak of tyrosine residues in BSA by PG. These results showed that PG interact with both tyrosine residues and tryptophan residues of BSA, which revealed in the molecular conformation of BSA changed, and furthermore led to the microenvironment of amino acid residue changed.

\section{Conclusion}

This paper presented spectroscopic studying on the interaction of PG with BSA using UV/vis spectrum, fluorescence emission spectrum, synchronous fluorescence spectrum, FT-IR spectrum and circular dichroism spectrum. The UV/vis absorption studies showed that BSA had a strong absorption peak at $278 \mathrm{~nm}$, which demonstrated the generation of the interaction between PG and aromatic amino acid residues of BSA. It was shown that the fluorescence of BSA has been quenched for reacting with PG and the quenching belonged to combined quenching type (both static and dynamic). In addition, the synchronous fluorescence spectrum and CD spectrum both indicated that the conformation of BSA molecule 
was changed with different concentration PG. All the spectroscopic results confirmed that a complex can form with PG binding to BSA molecule.

\section{References}

[1] G. Acampora and J. Hermans, Reversible denaturation of sperm whale myoglobin. I. Dependence on temperature, PH, and composition, J. Am. Chem. Soc. 89(7) (1967), 1543-1547.

[2] V. Anbazhagan and R. Renganathan, Study on the binding of 2,3-diazabicyclo [2.2.2] oct-2-ene with bovine serum albumin by fluorescence spectroscopy, Journal of Luminescence 128 (2008), 1454-1458.

[3] J.W. Bennett and R. Bentley, Seeing red: the story of prodigiosin, Advances in Applied Microbiology 47 (2000), 1-32.

[4] N. Brandes, P.B. Welzel, C. Werner and L.W. Kroh, Adsorption-induced conformational changes of proteins onto ceramic particles: differential scanning calorimetry and FTIR analysis, J. Colloid Interface Sci. 299 (2006), 56-69.

[5] M. Carbonaro, P. Maselli, P. Dore and A. Nucara, Application of Fourier transform infrared spectroscopy to legume seed flour analysis, Food Chem. 108 (2008), 361-368.

[6] Z.J. Cheng and Y.T. Zhang, Fluorometric investigation on the interaction of oleanolic acid with bovine serum albumin, J. Mol. Struct. 879 (2008), 81-87.

[7] R.W. Congdon, G.W. Muth and A.G. Splittgerber, The binding interaction of coomassie blue with proteins, Analytical Biochemistry 213 (1993), 407-413.

[8] C. Diaz-Ruiz, B. Montaner and R. Perez-Tomas, Prodigiosin induces cell death and morphological changes indicative of apoptosis in gastri cancer cell line HGT-1, Histology and histopathology 16(2) (2001), 415-421.

[9] G.D. Fasman, Circular Dichroism and Conformational Analysis of Biomolecules, Plenum Press, New York, 1996.

[10] L.N. Geng, X. Wang, N. Li, M.H. Xiang and K.A. Li, Characterization of hemoglobin immobilized on $\gamma$-zirconium phosphate, Colloids Surf. B: Biointerfaces 34 (2004), 231-238.

[11] N.J. Greenfield and G.D. Fasman, Computed circular dichroism spectra for the evaluation of protein conformation, Biochemistry 8 (1969), 4108-4116.

[12] F. Grimont and P. Grimon, The genus Serratia, The Prokaryotes 3 (2003), 219-244.

[13] Y.J. Hu, Y. Liu, Z.B. Pi and S.S. Qu, Interaction of cromolyn sodium with human serum albumin: a fluorescence quenching study, Bioorganic and Medicinal Chemistry 13 (2005), 6609-6614.

[14] M. Jackson and H.H. Mantsch, Protein Ligand Interactions: Structure and Spectroscopy, Oxford Univ. Press, New York, 2001.

[15] J.C. Kraak, S. Busch and H. Poppe, Study of protein-drug binding using capillary zone electrophoresis, Journal of Chromatography A 608 (1992), 257-264.

[16] C.V. Kumar, A. Buranaprapuk, G.J. Opiteck, M.B. Moyer, S. Jockusch and N.J. Turro, Photochemical protease: sitespecific photocleavage of hen egg lysozyme and bovine serum albumin, Proc. Natl. Acad. Sci. USA 95 (1998), 1036110366.

[17] B. Liu, D. Wei and S. Lu, Algicidal activity of prodigiosin against harmful algae, China Environmental Science 30(4) (2012), 477-482.

[18] Y. Liu, M.X. Xie, J. Kang and D. Zheng, Studies on the interaction of total saponins of Panax notoginseng and human serum albumin by Fourier transform infrared spectroscopy, Spectrochimica Acta Part A: Molecular and Biomolecular Spectroscopy 59 (2003), 2747-2758.

[19] J.B.F. Lloyd, Synchronized excitation of fluorescence emission spectra, Nature Phys. Sci. 231 (1971), 64-65.

[20] M. Mahato, P. Pal, T. Kamilya, R. Sarkar and G.B. Talapatra, Hemoglobin-silver interaction and bioconjugate formation: a spectroscopic study, J. Phys. Chem. B 114 (2010), 7062-7070.

[21] H.H. Mantsch and D. Chapman, Infrared Spectroscopy of Biomolecules, Wiley, New York, 1996.

[22] M.S. Melvin, K.E. Wooton, C.C. Rich, G.R. Saluta, G.L. Kucera, N. Lindquist and R.A. Manderville, Copper-nuclease efficiency correlates with cytotoxicity for the 4-methoxypyrrolic natural products, Journal of Inorganic Biochemistry 87(3) (2001), 129-135.

[23] P. Pal, T. Kamilya, M. Mahato and G.B. Talapatra, The formation of pepsin monomolecular layer by the Langmuirblodgett film deposition technique, Colloids Surf. B: Biointerfaces 73 (2009), 122-131.

[24] T. Peters, All about Albumin: Biochemistry, Genetics, and Medicinal Applications, Academic Press, San Diego, CA, 1996.

[25] J.M. Ruso, D. Attwood, M. Garcia, P. Taboada, L.M. Varela and V. Mosquera. A study of the interaction of the amphiphilic penicillins cloxacillin and dicloxacillin with human serum albumin in aqueous solution, Langmuir 17 (2001), 5189-5195.

[26] H. Slater, M. Crow, L. Everson and G.P. Salmond, Phosphate availability regulates biosynthesis of two antibiotics, prodigiosin and carbapenem, in Serratia via both quorum-sensing-dependent and -independent pathways, Molecular Microbiology 47(2) (2003), 303-320. 
[27] R.P. Williams, C.L. Gott, S.M. Qadri and R.H. Scott, Influence of temperature of incubation and type of growth medium on pigmentation in Serratia marcescens, J. Bacteriol. 106(2) (1971), 438-443.

[28] H. Yuan and J. Pawliszyn, Application of solid-phase microextraction in the determination of diazepam binding to human serum albumin, Analytical Chemistry 73 (2001), 4410-4416.

[29] T. Yuan, A.M. Weljie and H.J. Vogel, Tryptophan fluorescence quenching by methionine and selenomethionine residues of calmodulin: orientation of peptide and protein binding, Biochemistry 37 (1998), 3187-3195.

[30] L. Zhang, W.T. Liu, R.W. Zhang, Z.W. Wang, Z.D. Shen, X.H. Chen and K.S. Bi, Pharmacokinetic study of matrine, oxymatrine and oxysophocarpine in rat plasma after oral administration of Sophora flavescens Ait. extract by liquid chromatography tandem mass spectrometry, Journal of Pharmaceutical and Biomedical Analysis 47 (2008), 892-898.

[31] Y.Z. Zhang, J. Dai, X.P. Zhang, X. Yang, Y. Liu, Studies of the interaction between Sudan I and bovine serum albumin by spectroscopic methods, J. Mol. Struct. 888 (2008), 152-159. 


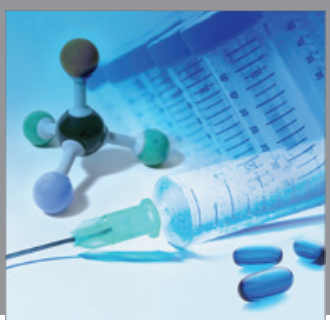

International Journal of

Medicinal Chemistry

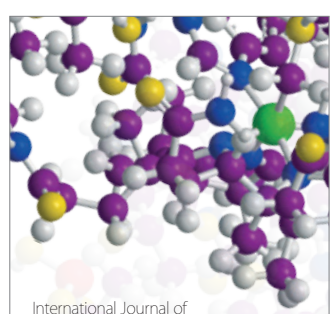

Carbohydrate Chemistry

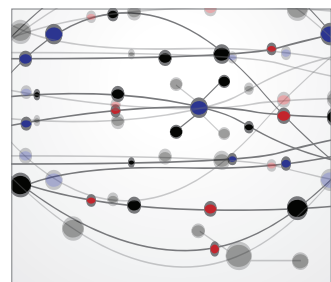

The Scientific World Journal
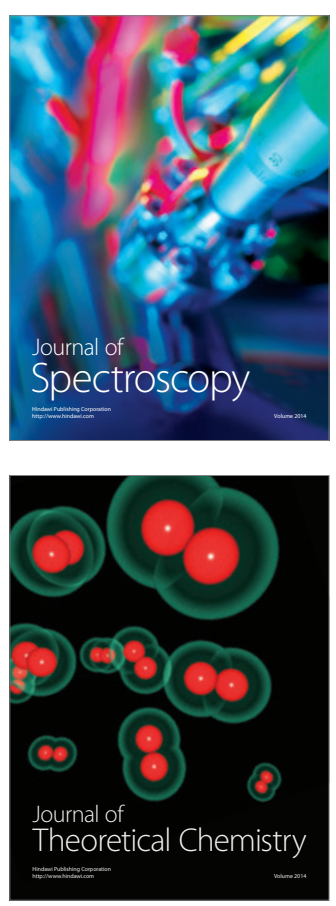
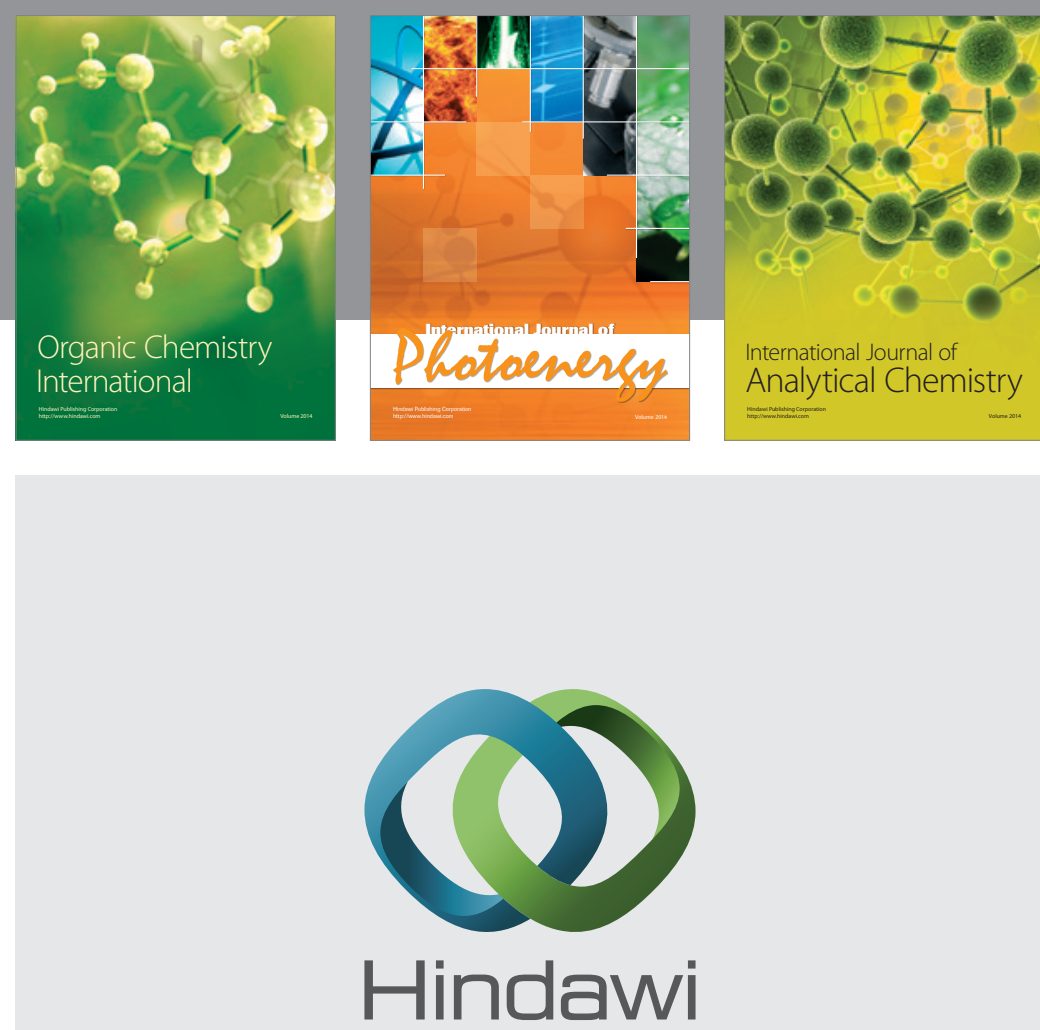

Submit your manuscripts at

http://www.hindawi.com
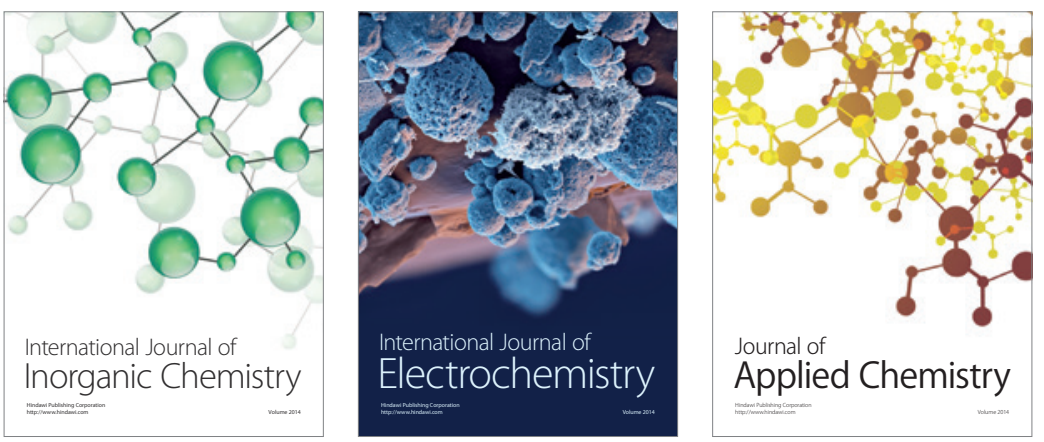

Journal of

Applied Chemistry
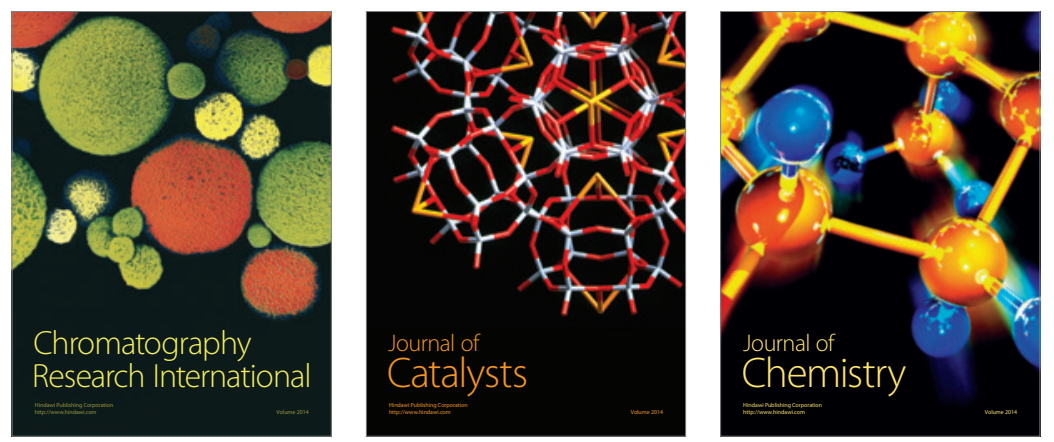
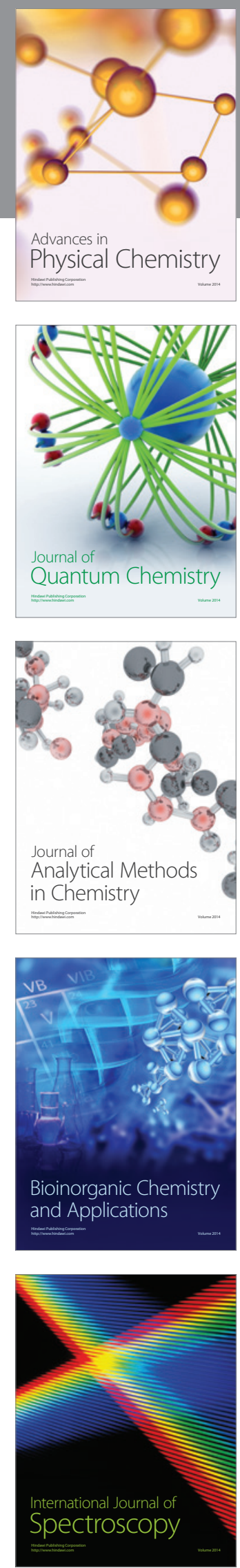